\title{
Invarianza Factorial de la Escala de Ansiedad Asociada a la Imagen Corporal en Universitarios Hombres y Mujeres
}

\author{
María del C. Zueck(1), Luis H. Blanco(2), Martha Ornelas ${ }^{(1)}$, Gabriel Gastélum(1)* \\ (1) Universidad Autónoma de Chihuahua. Facultad de Ciencias de la Cultura Física. DES Salud CAs 101 y \\ 121. Calle Escorza 900, CP 31000 Chihuahua, Chih.-México. (e-mail: mzueck@uach.mx, \\ mornelas@uach.mx; gastelum@uach.mx) \\ (2) Subdirección General de Enseñanza e Investigación del Hospital Civil de Guadalajara, Unidad \\ Hospitalaria "Fray Antonio Alcalde". Calle Hospital 278, Co. El Retiro Guadalajara, Jal.-México. \\ (e-mail: mdluishblanco01@gmail.com)
}

* Autor a quien debe enviarse la correspondencia.

Recibido Feb. 13, 2015; Aceptado Abr. 1, 2015; Versión final May. 2, 2015, Publicado Oct. 2015

\begin{abstract}
Resumen
El presente estudio analiza las propiedades psicométricas de la prueba Escala de Ansiedad Asociada a la Imagen Corporal (Body Image Anxiety Scale) versión rasgo en hombres y mujeres universitarios. La muestra total fue de 1545 participantes; 616 mujeres y 929 hombres, con una edad media de 18.11 años y 18.27 años, respectivamente. Los análisis factorial confirmatorios mostraron que una estructura bifactorial (promoción de la cultura e identidad cultural) es adecuada para ambas poblaciones (mujeres y hombres) de acuerdo a los requisitos psicométricos establecidos cuando los informantes son los propios alumnos. Además, la estructura factorial, las cargas factoriales y los interceptos se consideran invariantes en las dos poblaciones. Sin embargo, existen diferencias entre las poblaciones para las medias de los factores peso y no-peso.
\end{abstract}

Palabras clave: ecuaciones estructurales, estructura factorial, invarianza de medida, análisis factorial confirmatorio

\section{Factorial Invariance of the Body Image Anxiety Scale in Male and Female University Students}

\begin{abstract}
The present study analyses the psychometric properties of the trait version of Body Image Anxiety Scale applied to male and female university students. The overall sample consisted of 1545 subjects: 616 women and 929 men, with a mean age of 18.11 years and 18.27 years respectively. Psychometric analysis showed that a two-factorial structure (promotion of the culture and cultural identity) was adequate for both populations (men and women) according to the established psychometric requirements when the informers are the students themselves. The results showed that factor structure, factor loadings and intercepts of the instrument could be considered invariant across groups. However, there are differences between groups for the means of the weight and no-weight factors.
\end{abstract}

Keywords: structural equation, factor structure, measurement invariance, confirmatory factor analysis 


\section{INTRODUCCIÓN}

El interés por la delgadez y las preocupaciones por el peso vienen de un ideal cultural que aunque hoy sea considerado estético, es sólo una moda y no es necesariamente saludable, ni accesible, pudiendo tener consecuencias negativas, generadoras de ansiedad, como una gran preocupación por el peso y la figura, que pueden ser manifestadas como insatisfacción corporal, apreciada como el grado en que los individuos valoran o desprecian su cuerpo y/o en distorsión de la imagen corporal, que es la imprecisión en la determinación del tamaño corporal (Anuel et al., 2012). La imagen corporal y las normas estéticas que rigen actualmente al mundo occidental pueden afectar el desarrollo psicológico tanto de hombres como de mujeres, pero son las mujeres, preadolescentes y adolescentes, las que presentan una mayor tendencia a padecer conflictos en la elaboración de la imagen corporal, vinculados al desarrollo de problemas en las conductas alimentarias (Francisco et al., 2011; J. K. Thompson, 2003). Lo anterior se debe a que los estándares de "belleza y delgadez" son especialmente rígidos para ellas (Calaf et al., 2005).

La mayoría de los investigadores (Goñi y Rodríguez, 2007; Inglés et al., 2010; Lameiras et al., 2003; Solano y Cano, 2012; Wardle et al., 2006) de los trastornos de la conducta alimentaria concuerdan en que éstos tienen un origen multicausal, sobresaliendo la preocupación por la figura corporal y el seguimiento de dietas, sobre todo, en el sentido de pesar menos o adelgazar, estas conductas son consideradas de riesgo por los especialistas (Castillo, 2006; Inglés et al., 2010; Lameiras et al., 2003). Esta investigación se apoya en el estudio de la ansiedad desde la psicología, al utilizar herramientas de medición propias de esta área y aplicadas a población clínicamente sana, para conocer sus niveles de ansiedad estado y rasgo en su imagen corporal (Raich, 2000) mediante la aplicación de un instrumento de autoreporte que permite identificar la ansiedad que generalmente presentan las personas hacia áreas corporales que tienen que ver con su peso corporal, así como áreas relacionadas con la belleza física, que no tienen que ver con el peso intentando aportar evidencias y datos que propicien la intervención educativa dentro de una perspectiva de atención a la diversidad.

El presente estudio instrumental (Montero y León, 2005) se dirige a proporcionar apoyo empírico a la división factorial de prueba Body Image Anxiety Scale versión rasgo; lo que se justifica por la importancia de comprobar la estructura factorial de un instrumento y la equivalencia psicométrica del mismo en distintos grupos; ya que en el contexto de la comparación intergrupal, es indispensable plantearse la necesidad de llevar a cabo la adaptación de un instrumento de medida psicológica que cumpla con todos los criterios de equivalencia, pero sobre todo plantearse si la misma estructura factorial es aplicable a distintos grupos de sujetos (Abalo et al., 2006; Arbuckle, 2012). Por lo tanto, este trabajo pretende, por una parte, indagar si se replican los resultados psicométricos propuestos por Ornelas et al. (2014) para la prueba BIAS versión rasgo y, por otra, ampliarlos. Para ello, en primer lugar se comprobará el grado de congruencia de la estructura factorial del BIAS obtenida en el presente estudio y la reportada por (Ornelas et al., 2014). En segundo lugar, se calcula la invarianza factorial entre las muestras del presente estudio.

\section{METODOLOGÍA}

\section{Participantes}

La muestra de 1545 participantes y con un rango de edad de entre los 17 y 20 años, 616 mujeres (39.1\%, media 18.11 y desviación 0.69$)$ y 929 hombres $(60.1 \%$, media 18.27 y desviación 0.75$)$, se obtuvo mediante un muestreo por conveniencia, tratando de abarcar la representatividad de las diferentes licenciaturas que se ofrecen en la Universidad Autónoma de Chihuahua.

\section{Instrumento}

BIAS, versión rasgo propuesta por Ornelas et al. (2014) es una escala tipo Likert con 10 grados de intensidad., cuyo cuestionario se compone de 12 ítems, agrupados en dos factores (ansiedad peso y no peso) donde el encuestado responde, que tan ansioso(a), tenso(a) o nervioso(a) se siente con respecto a diferentes partes de su cuerpo.

\section{Procedimiento}

Se invitó a participar en el estudio a los estudiantes de primer ingreso de las licenciaturas que se ofrecen en la Universidad Autónoma de Chihuahua; los que aceptaron participar firmaron la carta de aceptación correspondiente. Luego se aplicó el instrumento utilizando el módulo administrador del instrumento (Ornelas et al., 2014), en una sesión de aproximadamente 15 minutos; en los centros de cómputo de las unidades académicas participantes. Al inicio de cada sesión se hizo una pequeña introducción sobre la importancia de la investigación. Las instrucciones de como responder se encontraban en las primeras pantallas. Al 
término de la sesión se les agradeció su participación. Una vez aplicado el instrumento se procedió a recopilar los resultados por medio del módulo generador de resultados del editor de escalas versión 2.0 (Blanco et al., 2013).

\section{Análisis de datos}

El análisis psicométrico se realizó en dos etapas: 1) análisis factorial confirmatorio y 2) análisis de invarianza factorial; con el fin de obtener una prueba que presente las mejores propiedades para la conformación de los puntajes de ansiedad. Para conducir el análisis factorial confirmatorio en cada muestra, se utilizó el software AMOS 21 (Arbuckle, 2012), las varianzas de los términos de error fueron especificados como parámetros libres, en cada variable latente (factor) se fijó uno de los coeficientes estructurales asociados a uno, para que su escala sea igual a la de una de las variables superficiales (ítems). El método de estimación empleado fue el de Máxima Verosimilitud; siguiendo la recomendación de B. Thompson (2004), en el sentido de que cuando se emplea análisis factorial confirmatorio se debe corroborar no sólo el ajuste de un modelo teórico sino que es recomendable comparar los índices de ajuste de varios modelos alternativos para seleccionar el mejor.

Para evaluar el ajuste del modelo se emplearon el estadístico Chi-cuadrado, el índice de bondad de ajuste (GFI) y el error cuadrático medio de aproximación (RMSEA) como medidas absolutas de ajuste. El índice de bondad ajustado (AGFI), el Índice Tucker-Lewis (TLI) y el índice de bondad de ajuste comparativo (CFI) como medidas de ajuste incremental. El índice de ajuste normado de parsimonia (PNFI), el índice de calidad de ajuste de parsimonia (PGFI), la razón de Chi-cuadrado sobre los grados de libertad (CMIN/GL) y el Criterio de Información de Akaike (AIC) como medidas de ajuste de parsimonia (Gelabert et al., 2011). Por último, se llevó a cabo un análisis de la invarianza factorial de los modelos de medida obtenidos, siguiendo las recomendaciones de Abalo et al. (2006), y se calculó la fiabilidad de cada una de las dimensiones a través del Alfa de Cronbach y el Coeficiente Omega (Revelle y Zinbarg, 2009).

\section{RESULTADOS}

\section{Análisis factorial confirmatorio}

De acuerdo a los resultados de la Tabla 1 el análisis factorial confirmatorio de 12 ítems agrupados en dos factores en la muestra de mujeres es aceptable (GFI .970 y RMSEA .050) y de acuerdo a las medidas de ajuste incremental y de parsimonia significativamente superior al modelo independiente y muy similar al modelo saturado. En la Tabla 1, GFI es el índice de bondad de ajuste; RMSEA es la raíz del error medio; AGFI es el índice corregido de la bondad de ajuste; TLI es el índice de Tucker-Lewis; CFI es el índice de ajuste comparativo; CMIN/DF es el índice de ajuste chi cuadrado dividido por los grados de libertad; y AIC es el criterio de información de Akaike.

Por otro lado, el análisis factorial confirmatorio en la muestra de hombres (Tabla 1), indica que el modelo de medición de dos factores es también aceptable (GFI .971 y RMSEA .054) y de acuerdo a las medidas de ajuste incremental y de parsimonia significativamente superior al modelo independiente y muy similar al modelo saturado.

Tabla 1: Índices absolutos, incrementales y de parsimonia para los modelos generados. Análisis factorial confirmatorio para mujeres y hombres (* $p<.05)$.

\begin{tabular}{|c|c|c|c|c|c|c|c|c|}
\hline \multirow[b]{2}{*}{ Modelo } & \multicolumn{3}{|c|}{ Índices absolutos } & \multicolumn{3}{|c|}{ Índices incrementales } & \multicolumn{2}{|c|}{ Índices de parsimonia } \\
\hline & $\chi^{2}$ & GFI & RMSEA & AGFI & TLI & $\mathrm{CFI}$ & CMIN/DF & $\mathrm{AIC}$ \\
\hline \multicolumn{9}{|c|}{ Solución factorial para los mujeres } \\
\hline Independiente & $4241.907^{*}$ & .320 & .321 & .197 & .000 & .000 & 64.271 & 4265.907 \\
\hline Saturado & 0.000 & 1.000 & & & & 1.000 & & 156.000 \\
\hline 2 factores & $118.750^{*}$ & .970 & .050 & .949 & .976 & .983 & 2.527 & 180.750 \\
\hline \multicolumn{9}{|c|}{ Solución factorial para las hombres } \\
\hline Independiente & $7761.721^{*}$ & .237 & .354 & .098 & .000 & .000 & 117.602 & 7785.721 \\
\hline Saturado & 0.000 & 1.000 & & & & & & 156.000 \\
\hline 2 factores & $173.350^{*}$ & .971 & .054 & .952 & .977 & .984 & 3.688 & 235.350 \\
\hline
\end{tabular}

De acuerdo a los resultados de la Tabla 2, en ambas muestras, todos los ítems saturan adecuadamente en su dimensión (factor) prevista. Observándose correlaciones moderadamente altas entre los factores evidenciando una no muy buena validez discriminante. 
Tabla 2: Soluciones estandarizadas para el análisis factorial confirmatorio en ambas muestras.

\begin{tabular}{|c|c|c|c|c|}
\hline \multicolumn{5}{|c|}{ Pesos factoriales } \\
\hline & \multicolumn{2}{|c|}{ Factor Peso } & \multicolumn{2}{|c|}{ Factor No-Peso } \\
\hline Ítem & mujeres & hombres & mujeres & hombres \\
\hline Piernas & .84 & .84 & & \\
\hline Muslos & .81 & .78 & & \\
\hline Caderas & .81 & .71 & & \\
\hline Nalgas & .77 & .78 & & \\
\hline Abdomen & .62 & .71 & & \\
\hline Cintura & .62 & .69 & & \\
\hline Labios & & & .80 & .67 \\
\hline Frente & & & .77 & .81 \\
\hline Manos & & & .72 & .79 \\
\hline Cuello & & & .72 & .89 \\
\hline Mentón & & & .71 & .84 \\
\hline Muñecas & & & .70 & .70 \\
\hline \multicolumn{5}{|c|}{ Correlaciones entre factores } \\
\hline Factor Peso & - & - & & \\
\hline Factor No-Peso & .50 & .73 & - & - \\
\hline
\end{tabular}

Invarianza de la estructura factorial entre mujeres y hombres universitarios

Los índices de ajuste obtenidos (Tabla 3) permiten aceptar la equivalencia de los modelos de medida básicos entre las dos muestras. Aunque el valor de Chi-cuadrado excede al exigido para aceptar la hipótesis de invarianza, el resto de índices contradicen esta conclusión (GFI .970; CFI .994; RMSEA .037; AIC 416.101) lo que nos permite aceptar el modelo base de la invarianza (modelo sin restricciones). En la Tabla 3, GFI es el índice de bondad de ajuste; NFI es el índice de ajuste normado; CFI es el índice de ajuste comparativo; RMSEA es la raíz del error medio; y AIC es el criterio de Información de Akaike.

Añadiendo al modelo base restricciones sobre las cargas factoriales caracterizamos la invarianza métrica. Los valores que se recogen en la tabla 3 permiten aceptar este nivel de invarianza. El índice de ajuste general $(\mathrm{GFI}=.965)$ y el error cuadrático medio de aproximación $(\mathrm{RMSEA}=.039)$ siguen aportando información convergente en esta dirección. Además, el criterio de información de Akaike $(\mathrm{AIC}=454.388)$ y el índice comparativo de Bentler $(\mathrm{CFI}=.979)$ no sufren grandes variaciones respecto al modelo anterior. Haciendo uso del criterio para la evaluación de los modelos anidados propuesto por Cheung y Rensvold (2002), quiénes sugieren que si el cálculo de la diferencia de los CFI de ambos modelos anidados disminuye en .01 o menos, se da por bueno el modelo restringido y por tanto el cumplimiento de la invarianza factorial; la diferencia entre CFls obtenida (.004) permite aceptar el modelo de invarianza métrica. Podemos concluir hasta ahora que las cargas factoriales son equivalentes en las dos muestras.

Una vez demostrada la invarianza métrica entre las muestras, pasamos a evaluar la equivalencia entre interceptos (invarianza factorial fuerte). Los índices (Tabla 3) muestran un buen ajuste de este modelo, tanto evaluado de modo independiente como analizándolo respecto a su anidamiento con el modelo de invarianza métrica. La diferencia entre los índices comparativos de Bentler es de .008; el índice de ajuste general es 0.956 y el error cuadrático medio de aproximación es .045. Aceptada la invarianza fuerte, los dos modelos evaluados son equivalentes respecto a los coeficientes factoriales y a los interceptos.

Tabla 3 Índices de bondad de ajuste de cada uno de los modelos puestos a prueba en la invarianza factorial $\left({ }^{*} p<.05 ;\right)$

\begin{tabular}{|l|c|c|c|c|c|c|c|}
\hline \multicolumn{1}{|c|}{ Modelo } & \multicolumn{7}{c|}{ Índice de Ajuste } \\
\hline & $\chi^{2}$ & gl & GFI & NFI & CFI & RMSEA & AIC \\
\hline Modelo sin restricciones & $292.101^{*}$ & 94 & .970 & .976 & .983 & .037 & 416.101 \\
\hline Invarianza métrica & $350.388^{*}$ & 104 & .965 & .971 & .979 & .039 & 454.388 \\
\hline Invarianza factorial fuerte & $445.795^{*}$ & 107 & .956 & .963 & .971 & .045 & 543.795 \\
\hline
\end{tabular}


Los factores obtenidos en los análisis factoriales confirmatorios alcanzan valores de consistencia interna por encima de .75 en ambas muestras (hombres y mujeres); evidenciando una consistencia interna adecuada para este tipo de subescalas, particularmente si se considera el número reducido de ítems (Tabla 4).

Tabla 4 Coeficiente omega y alfa para los factores obtenidos.

\begin{tabular}{|l|c|c|c|c|}
\hline & \multicolumn{2}{|c|}{ Mujeres } & \multicolumn{2}{c|}{ Hombres } \\
\hline Factor & $\Omega$ & $\alpha$ & $\Omega$ & $\alpha$ \\
\hline 1. Peso & .884 & .896 & .886 & .873 \\
\hline 2. No-Peso & .753 & .897 & .853 & .920 \\
\hline
\end{tabular}

Contrastes de las medias de los factores entre mujeres y hombres

Una vez comprobada la invarianza factorial, las diferencias entre las medias de los factores de los dos grupos se estimaron tomando como referente la muestra de hombres, fijando en 0 el valor de las medias para dicha muestra y estimando libremente el valor de las medias para la muestra de mujeres. Las restricciones sobre los coeficientes de regresión e interceptos, requeridos para los contrastes entre las medias se realizaron automáticamente mediante el software AMOS 21 (Arbuckle, 2012). Los resultados de las comparaciones entre medias indicaron que la media del factor peso fue significativamente mayor $(0.933$, $p<0.001)$ en las mujeres y en el factor no-peso $(-0.539, p<0.001)$ significativamente mayor en los hombres.

\section{CONCLUSIONES}

De los resultados mostrados, de su análisis y de su discusión, y tomando en cuenta que el objetivo principal de este estudio fue el de examinar la estructura factorial y la medición de la invariancia de dicha estructura en alumnos y alumnas universitarios, se pueden obtener las siguientes conclusiones:

1) El Análisis Factorial Confirmatorio, en ambas muestras, indicó que el ajuste de los datos al modelo teórico de 12 ítems agrupados en dos factores es aceptable. Al mismo tiempo que los dos factores así obtenidos presentan en general saturaciones factoriales estandarizadas adecuadas. Por su parte los factores correlacionan entre sí de forma positiva y estadísticamente significativa lo cual muestra que a medida que aumenta la ansiedad percibida en alguno de los factores, también aumenta en el otro.

2) Los factores en ambas muestras evidenciaron una consistencia interna adecuada, particularmente si se considera el número reducido de ítems en cada uno de ellos.

3) Conjuntamente con todo lo antes dicho, los resultados del análisis de la invarianza factorial entre las muestras; indican una alta congruencia entre pares de factores. Lo que sugiere la existencia de fuertes evidencias de la validación cruzada de la medida y por tanto de la estabilidad de la estructura, hasta que no se demuestre lo contrario.

4) Las comparaciones entre los grupos reflejaron diferencias significativas de mayor ansiedad en las mujeres en el factor peso y de mayor ansiedad para los hombres en el factor no-peso. Lo que parece indicar que las mujeres se preocupan más por los aspectos relacionados con su peso y los hombres por los relacionados con los de no peso.

En síntesis, el análisis de las propiedades psicométricas, ha mostrado que una estructura bifactorial es viable y apropiada de acuerdo a los requisitos psicométricos establecidos cuando los informantes son los propios alumnos. La estructura de dos factores, atendiendo a criterios estadísticos y sustantivos, ha mostrado adecuados indicadores de ajuste, de fiabilidad y de validez. Sin embargo, consideramos que más estudios son necesarios con el fin de corroborar o refutar los datos obtenidos en la presente investigación. Dos limitaciones están presentes en este trabajo. La primera es que los participantes son solo estudiantes universitarios mexicanos, lo que supone una amenaza para la posibilidad de generalizar estos resultados. Ampliar la muestra (agregando por ejemplo adultos jóvenes que no son estudiantes) es un área de trabajo de cara al futuro. La segunda limitación proviene del propio instrumento de evaluación, que se basa en el autoinforme y que puede contener los sesgos que se derivan de la deseabilidad social.

\section{REFERENCIAS}

Abalo J., Lévy J., Rial A. y Varela J., Invarianza factorial con muestras múltiples, En Modelización con Estructuras de Covarianzas en Ciencias Sociales por J. Lévy, pp 259-278 Netbiblo, Madrid, (2006) 
Anuel A., Bracho A., Brito N., Rondón J. E. y Sulbarán D., Autoaceptación y mecanismos cognitivos sobre la imagen corporal, Psicothema, 24(3), 390-395, (2012)

Arbuckle J. R., AMOS users guide version 21.0, Marketing Department, SPSS Incorporated, Chicago, IL, (2012)

Blanco H., Ornelas M., Tristán J. L., Cocca A., Mayorga-Vega D., López-Walle J., et al., Editor for creating and applying computerise surveys, doi: http://dx.doi.org/10.1016/j.sbspro.2013.12.105, Procedia Social and Behavioral Sciences, 106, 935-940, (2013)

Calaf M., León M., Hilerio C. y Rodríguez J., Inventario de Imagen Corporal para Féminas Adolescentes (IICFA), Revista Interamericana de psicología, 39(3), 347-354, (2005)

Castillo B., Sociedad de consumo y trastornos de la conducta alimentaria, Trastornos de la Conducta Alimentaria, 4, 321-335, (2006)

Cheung G. W. y Rensvold R. B., Evaluating goodness-of-fit indexes for testing measurement invariance, doi: 10.1207/s15328007SEM0902_5, Structural Equation Modeling, 9(2), 233-255, (2002)

Francisco R., Alarcão M. y Narciso I., Avaliação de factores de risco de desenvolvimento de perturbações alimentares: Desenvolvimento e estudos de validação da versão portuguesa do McKnight Risk Factor Survey IV, Revista Iberoamericana de Diagnóstico y Evaluación Psicológica, 32(2), 143-170, (2011)

Gelabert E., García-Esteve L., Martín-Santos R., Gutiérrez F., Torres A. y Subirà S., Psychometric properties of the Spanish version of the Frost Multidimensional Perfectionism Scale in women, Psicothema, 23(1), 133139, (2011)

Goñi A. y Rodríguez A., Variables associated with the risk for eating disorders in adolescence, Salud Mental, 30(4), 16-23, (2007)

Inglés C. J., Piqueras J. A., García-Fernández J. M., García-López L. J., Delgado B. y Ruiz-Esteban C., Diferencias de género y edad en respuestas cognitivas, psicofisiológicas y motoras de ansiedad social en la adolescencia, Psicothema, 22(3), 376-381, (2010)

Lameiras M., Calado M., Rodríguez Y. y Fernández M., Hábitos alimentarios e imagen corporal en estudiantes universitarios sin trastornos alimentarios, Revista Internacional de Psicología Clínica y de la Salud, 3(1), 23-33, (2003)

Montero I. y León O., Sistema de clasificación del método en los informes de investigación en Psicología, International Journal of Clinical and Health Psychology, 5, 115-127, (2005)

Ornelas M., Gastélum G., Blanco H. y Peinado J. E., Análisis psicométrico de la prueba Body Image Anxiety Scale Versión Rasgo en alumnos de educación superior, Perfiles Educativos, 36(144), 120-137, (2014)

Raich R. M., Imagen corporal. Conocer y valorar el propio cuerpo, Pirámide, Madrid, (2000)

Revelle W. y Zinbarg R. E., Coefficients alpha, beta, omega and the glb: comments on Sijtsma, doi: 10.1007/s11336-008-9102-z, Psychometrika, 74(1), 145-154, (2009)

Solano N. y Cano A., Ansiedad en los trastornos alimentarios: Un estudio comparativo, Psicothema, 24(3), 384-389, (2012)

Thompson B., Exploratory and Confirmatory Factor Analysis. Understanding concepts and applications. , American Psychological Association, Washington, D C, (2004)

Thompson J. K., Introduction: Body image, eating disorders, and obesity - An emerging synthesis, En Body image, eating disorders, and obesity: An Integrative Guide for Assessment and Treatment, por J. K. Thompson, pp 1-20 American Psychological Association, Washington, D C, (2003)

Wardle J., Haase A. y Steptoe A., Body image and weight control in young adults: International comparisons in university students from 22 countries, International Journal of Obesity, 30, 644-651, (2006) 